\title{
Clinical implication of preoperative psoas muscle area in endometrial cancer patients
}

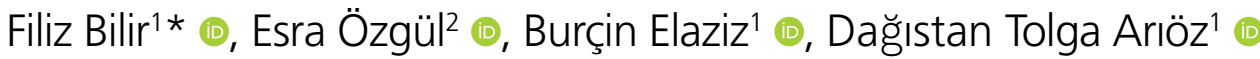

\section{SUMMARY}

OBJECTIVE: Obesity is a significant risk factor for endometrial cancer. In contrast, sarcopenia describes a loss of the body's muscle mass that is closely related to unfavorable clinical outcomes. Even endometrial cancer patients have high rates of obesity, and they should have a significantly higher risk for undiagnosed sarcopenia or fragile muscle quality.

METHODS: This is a retrospective study that included an endometrial cancer database collected from a tertiary gynecologic cancer center. We investigated the relationship between preoperative psoas muscle area by magnetic resonance imaging, surgical outcomes and pathological features.

RESULTS: The study included 116 patients, the mean height was $160 \mathrm{~cm}$ (Standart deviation 7), weight was $72 \mathrm{~kg}$ (Standart deviation 18), and the median duration of hospitalization was 4 days (Interquartile range 2-9) in the whole study group. Sarcopenia was diagnosed in $25(21.6 \%)$ patients, according to the magnetic resonance imaging findings. Three (6.5\%) obese patients had sarcopenia, but it was $31.4 \%$ in nonobese patients ( $p=0.026)$. The median duration of hospitalization was five days (3-9 days) in the sarcopenia group, and it was four days (2-7 days) in the non-sarcopenia group.

CONCLUSION: Sarcopenic patients did not have increased surgical complication rates following uterine cancer surgery. We should be aware of hospitalization duration in those patients, and sarcopenic counterparts necessitate longer follow-up after the surgery. KEYWORDS: Endometrial cancer. Sarcopenia. Stay lengths. Histopathology.

\section{INTRODUCTION}

The most common gynecologic cancer is endometrial cancer in women, and more than 10,000 deaths are expected in the USA per year ${ }^{1}$. Many reproductive risk factors such as early age of menarche and elder age of menopause, nulliparity, infertility, the first age of delivery elder than 30 years old, and drugs such as tamoxifen for breast cancer prevention have been associated with a higher risk of developing endometrial cancer ${ }^{2}$. Also, obesity is a significant risk factor for endometrial cancer, and it has an increased relative risk in the disease incidence at least threefold ${ }^{1}$. In addition to the cancer risk, obesity causes poorer oncological outcomes than normal-weight patients ${ }^{3}$.
Sarcopenia describes a loss of the body's muscle mass that can cause chronic disease, cancer, and advanced age and is closely related to unfavorable clinical outcomes ${ }^{4}$. In sarcopenia, skeletal muscle changes can exist independently from body mass index (BMI) and usually cannot be clinically diagnosed, especially in obese cancer patients 5 . Decreased muscle mass has been linked to poorer oncological outcomes related to radiodensity and is called myosteatosis. Also, it is associated with worse surgical complications $s^{6}$. High rates of obesity in endometrial cancer patients can have a significantly higher risk for undiagnosed sarcopenia or fragile muscle quality. It may be associated with poorer outcomes. That is why we want to investigate sarcopenia and its possible clinical implications in endometrial cancer patients with FIGO stages I-III.

\footnotetext{
${ }^{1}$ Afyonkarahisar Health Science University, Department of Gynecologic Oncology - Afyonkarahisar, Turkey.

${ }^{2}$ Afyonkarahisar Health Science University, Department of Radiology - Afyonkarahisar, Turkey.

*Corresponding author: drflzyldz@hotmail.com

Conflicts of interest: the authors declare there are no conflicts of interest. Funding: none.

Received on April 03, 2021. Accepted on May 19, 2021.
} 


\section{METHODS}

\section{Patient selection}

Following the local institutional review board's approval, all medical records of patients with endometrial cancer, proven histologically, were reviewed. Patients who had evidence of distant disease in preoperative imaging were excluded from the study. We recorded demographic features such as age, stage, baseline laboratory parameters, BMI, weight, and magnetic resonance imaging (MRI) findings for sarcopenia from the hospital records. The surgical procedure included total hysterectomy, omentectomy, peritoneal washing, and lymph node dissection if needed. After the surgery, all patients were referred to medical and radiation oncology clinics for adjuvant treatment planning. BMI was calculated with the following formula: the patient's weight $(\mathrm{kg})$ divided by height (m) squared. According to the National Institutes of Health $(\mathrm{NIH})$ criteria, all patients were grouped as obese $(\mathrm{BMI}>30)$ and nonobese $(\mathrm{BMI}<30)^{7}$.

\section{Psoas muscle area measurement}

Patients who underwent preoperative upper abdominal MRI were included in the study. The study included 116 patients, and an abdominal MRI was performed in a preoperative setting. MRI was performed with a $1.5 \mathrm{~T}$ (Siemens, Magnetom Aera, Erlangen, Germany) unit with a 4-channeled body coil. Preoperative axial MR images of the patients were transferred to the open-access software Image J program and evaluated by a 12-year-experienced abdominal radiologist. The psoas muscle area (PMA) was calculated in the sections passing through the L3 vertebral body where both transverse processes were seen. The right and left psoas muscle borders were drawn manually by the same radiologist (Figure 1). The areas of the right and left psoas muscles were calculated separately with the Image J program and summed. Based on data published by Golse et al. ${ }^{8}$ in 2017 and Farkas et al. ${ }^{9}$ in 2019, sarcopenia was defined as a PMA $<1561 \mathrm{~mm}^{2}$ for males and $<1464$ $\mathrm{mm}^{2}$ for females.

\section{Statistical analysis}

Demographics and pathological features were stratified as the groups with sarcopenia or normal weight by $\chi^{2} /$ Fisher's exact test for categorical data and Student's $t$-test for continuous data (Table 1).

A normality test was performed for all data and determined with standard deviation or interquartile range as appropriate. All analyses were done with SPSS version 22.0 (IBM Corporation, New York, USA). $\mathrm{p}<0.05$ were considered statistically significant.

\section{RESULTS}

The whole study group's mean age was 61.7 years old (sd 10), and the BMI was 28 (SD 7). The most common histological subtype was endometrioid adenocarcinoma in 103 (89\%) patients. The mean height was $160 \mathrm{~cm}$ (SD 7), the mean weight was $72 \mathrm{~kg}$ (SD 18), and the median duration of hospitalization was 4 days (IR 2-9) in the whole study group.

Total PMA was calculated as the sum of left and right PMA values. The median left PMA was $1420 \mathrm{~mm}^{2}$ (461-4659), the median right PMA was $1393 \mathrm{~mm}^{2}$ (452-4865), and the total PMA was $2867 \mathrm{~mm}^{2}$. Sarcopenia was diagnosed in 25 (21.6\%) patients, according to the MRI findings. Three (6.5\%) obese patients had sarcopenia, but it was $31.4 \%$ in nonobese patients (chi-square p-value 0.026). When we analyzed diabetic patients, they had a $31.7 \%$ sarcopenia prevalence, but nondiabetic patients had 16\% (p-value 0.002). The regression analysis included FIGO stage, myometrial, serosal, lymphovascular, perineural invasion, lymph node metastasis, and tumor grade, but it did not show a significant correlation with PMA measurements ( $\mathrm{p}$-values were $0.49,0.6,0.8,0.7,0.32,0.16$, and 0.3 , respectively) (Table 2).

Postoperative complications such as 90-day mortality, surgical site infection, ileus, urinary tract infection, and hospitalization duration were recorded in the study, and PMA values significantly correlated with hospitalization duration $(\mathrm{p}=0.001)$. The median duration of hospitalization was five days (3-9 days) in the sarcopenia group, and it was four days (2-7 days) in the non-sarcopenia group.

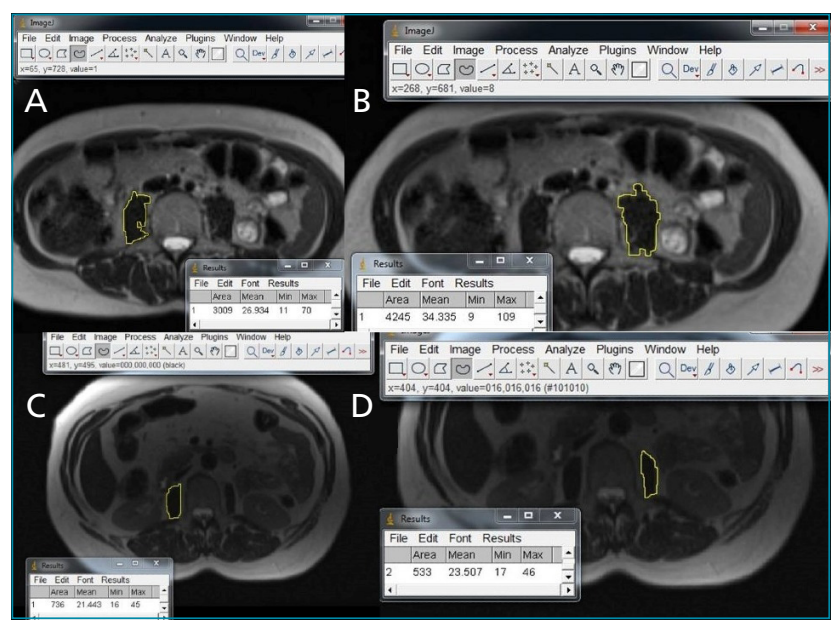

Figure 1. Manual psoas muscle area measurement is seen using preoperative magnetic resonance imaging scans. Measurement of right and left psoas muscle area are seen $(A, B)$ in a 43-year-old female patient's axial T2 magnetic resonance imaging scan. Her total psoas muscle area is within normal limits. (C, D) Sarcopenia is detected in a 58-year-old female patient's axial T2 magnetic resonance imaging scan. Her psoas muscle area is $1269 \mathrm{~mm}^{2}$ (less than $1464 \mathrm{~mm}^{2}$ ). 


\section{DISCUSSION}

This study investigated preoperative sarcopenia incidence with MRI and its possible correlation with the pathological features of endometrial cancer. Our results showed that many pathological parameters did not differ between sarcopenia and non-sarcopenia patients. Only one parameter, i.e., the duration of hospitalization, was significantly higher in the sarcopenia group.

Table 1. Pathological characteristics of the patients.

\begin{tabular}{|c|c|}
\hline $\begin{array}{l}\text { Pathological } \\
\text { characteristics }\end{array}$ & Number of patients (\%) \\
\hline \multicolumn{2}{|l|}{ FIGO stage } \\
\hline Stage I & $44(38)$ \\
\hline Stage II & $52(49)$ \\
\hline Stage III & $20(17)$ \\
\hline \multicolumn{2}{|c|}{ Myometrial invasion } \\
\hline Lower than $1 / 2$ & $61(53)$ \\
\hline Higher than $1 / 2$ & $55(47)$ \\
\hline \multicolumn{2}{|c|}{ Cervical stromal invasion } \\
\hline Negative & $102(88)$ \\
\hline Positive & $14(12)$ \\
\hline \multicolumn{2}{|c|}{ Lymphovascular invasion } \\
\hline Negative & $95(82)$ \\
\hline Positive & $21(18)$ \\
\hline \multicolumn{2}{|l|}{ Serosal invasion } \\
\hline Negative & $108(93)$ \\
\hline Positive & $8(7)$ \\
\hline \multicolumn{2}{|c|}{ Lymph node metastasis } \\
\hline Negative & $102(88)$ \\
\hline Positive & $14(12)$ \\
\hline \multicolumn{2}{|c|}{ Perineural invasion } \\
\hline Negative & $114(98)$ \\
\hline Positive & $2(2)$ \\
\hline \multicolumn{2}{|l|}{ Tumor grade } \\
\hline Grade I & $46(40)$ \\
\hline Grade II & $43(37)$ \\
\hline Grade III & $27(23)$ \\
\hline
\end{tabular}

Obesity is one of the common risk factors for endometrial cancer and causes nearly threefold relative risk. Otherwise, obesity affects the prognosis of endometrial cancer and poorer outcomes than normal-weight counterparts ${ }^{10}$. Sarcopenia is a different condition with muscle mass loss and/or decreased muscle quality. It negatively impacts cancer patients, including postoperative complications and lower chemotherapy tolerance ${ }^{11}$. Sarcopenia in pancreatic and lung

Table 2. Characteristics and anthropometrics of patients with endometrial cancer stratified by sarcopenia and non-sarcopenia.

\begin{tabular}{l|c|c|c}
$\begin{array}{l}\text { Pathological } \\
\text { characteristics }\end{array}$ & $\begin{array}{c}\text { Sarcopenia, } \\
n\end{array}$ & $\begin{array}{c}\text { Non- } \\
\text { sarcopenia, n }\end{array}$ & p-value \\
\hline
\end{tabular}

FIGO stage

\begin{tabular}{l|c|c|c}
\hline Stage I & 8 & 36 & 0.77 \\
\hline Stage II & 12 & 40 & \\
\hline Stage III & 5 & 15 & \\
\hline
\end{tabular}

Myometrial invasion

\begin{tabular}{l|l|l|l}
\hline Lower than $1 / 2$ & 12 & 49 & 0.6 \\
\hline Higher than $1 / 2$ & 13 & 42 & \\
\hline
\end{tabular}

Cervical stromal invasion

\begin{tabular}{l|c|c|c}
\hline Negative & 24 & 78 & 0.16 \\
\hline Positive & 1 & 13 & \\
\hline
\end{tabular}

Lymphovascular invasion

\begin{tabular}{l|c|c|c}
\hline Negative & 21 & 74 & 0.75 \\
\hline Positive & 4 & 17 & \\
\hline
\end{tabular}

\begin{tabular}{l|c|c|c|}
\hline \multicolumn{5}{l|}{ Serosal invasion } \\
\hline Negative & 23 & 85 & 0.8 \\
\hline Positive & 2 & 6 & \\
\hline
\end{tabular}

Lymph node metastasis

\begin{tabular}{l|c|c|c}
\hline Negative & 24 & 78 & 0.16 \\
\hline Positive & 1 & 13 & \\
\hline
\end{tabular}

Perineural invasion

\begin{tabular}{l|c|c|c}
\hline Negative & 24 & 90 & 0.32 \\
\hline Positive & 1 & 1 & \\
\hline
\end{tabular}

Tumor grade

\begin{tabular}{l|c|c|c}
\hline Grade I & 7 & 39 & 0.40 \\
\hline Grade II & 11 & 32 & \\
\hline Grade III & 7 & 20 & \\
\hline
\end{tabular}


cancer patients was broadly investigated in the literature. A recent study showed poorer overall survival in patients with pancreatic cancer who had sarcopenia. The study measured CT-based imaging and included a preoperative setting. Thereby, $90 \%$ of patients had early-stage pancreatic cancer 5 . Similar to our results, they did not find a significant correlation between sarcopenia and pathological parameters including tumor grade, lymphovascular invasion, and perineural invasion. Portal et al. found that sarcopenia has a significantly lower survival in patients diagnosed with lung cancer ${ }^{12}$. With respect to surgical complications, a meta-analysis revealed that preoperative sarcopenia is associated with prolonged hospital stay following pancreatic cancer surgery. Still, it did not have a negative impact on postoperative morbidity ${ }^{13}$.

There is scarce literature about sarcopenia in gynecologic cancers; even if most gynecologic cancer patients can be overweight, sarcopenia is not uncommon in this group. A review about sarcopenia incidence showed a wide range from 11 to $74 \%$ overall population and from 1 to $36 \%$ in the setting of obesity ${ }^{14}$. Moreover, sarcopenic visceral obesity is a different condition than sarcopenia, and it is characterized as increased visceral fat with decreased muscle mass. Further, it can cause higher postoperative mortality, surgical complications, and lower survival rates ${ }^{15,16}$. In our study, obese patients had a $6.5 \%$ of sarcopenia rate, which was concordant with the literature; on the other hand, they showed a similar postoperative complication rate compared with sarcopenic patients with normal weight. A few studies investigated sarcopenia in endometrial cancer, measuring sarcopenia with CT-based imaging, although they showed poorer oncological outcomes in sarcopenic patients, none of them investigated postoperative complications. Our study was the first MRI-based imaging research for sarcopenia and uterine cancer. The main result of our study was increased hospital stay in patients with sarcopenia.

Also, we have some major limitations such as deficiency of oncological outcomes as overall survival, disease survival, or recurrence rates; thereby, short follow-up period, we could not analyze these parameters. Actually, we did not aim because our focusing intent was postoperative complications.

\section{CONCLUSION}

Sarcopenic patients did not have increased surgical complication rates following uterine cancer surgery. We should be aware of hospitalization duration in those patients, and sarcopenic counterparts necessitate longer follow-up after the surgery.

\section{AUTHORS" CONTRIBUTIONS}

FB: Conceptualization, Writing - original draft. DTA: Data curation. EO: Formal Analysis. BE: Formal Analysis, Writing - review \& editing.

\section{REFERENCES}

1. Reeves GK, Pirie K, Beral V, Green J, Spencer E, Bull D, et al. Cancer incidence and mortality in relation to body mass index in the Million Women Study: cohort study. BMJ. 2007;335(7630):1134. https://doi.org/10.1136/ bmj.39367.495995.AE

2. Ali AT. Reproductive factors and the risk of endometrial cancer. Int J Gynecol Cancer. 2014;24(3):384-93. https://doi. org/10.1097/IGC.0000000000000075

3. Fearon K, Arends J, Baracos V. Understanding the mechanisms and treatment options in cancer cachexia. Nat Rev Clin Oncol. 2013;10(2):90-9. https://doi.org/10.1038/nrclinonc.2012.209

4. Shachar SS, Williams GR, Muss HB, Nishijima TF. Prognostic value of sarcopenia in adults with solid tumours: a metaanalysis and systematic review. Eur J Cancer. 2016;57:58-67. https://doi.org/10.1016/j.ejca.2015.12.030

5. Peng YC, Wu CH, Tien YW, Lu TP, Wang YH, Chen BB. Preoperative sarcopenia is associated with poor overall survival in pancreatic cancer patients following pancreaticoduodenectomy. Eur Radiol. 2021;31(4):2472-81. https://doi.org/10.1007/ s00330-020-07294-7

6. Dériaz $O$, Dumont $M$, Bergeron $N$, Després JP, Brochu $M$, Prud'homme D. Skeletal muscle low attenuation area and maximal fat oxidation rate during submaximal exercise in male obese individuals. Int J Obes Relat Metab Disord. 2001;25(11):1579-84. https://doi.org/10.1038/sj.ijo.0801809
7. Goodwin, S. The Practical guide to the Identification, evaluation and treatment of overweight and obesity in adults. Clin Nurse Spec. 2002;16(3):164. https://doi.org/10.1097/00002800200205000-00016

8. Golse N, Bucur PO, Ciacio O, Pittau G, Sa Cunha A, Adam R, et al. A new definition of sarcopenia in patients with cirrhosis undergoing liver transplantation. Liver Transpl. 2017;23(2):14354. https://doi.org/10.1002//t.24671

9. Farkas ZC, Rashid T, Chen YS, Siddiqui TM, Yandrapalli S, Frager S, et al. The correlation between sarcopaenia and post-transjugular intrahepatic portosystemic shunt hepatic encephalopathy: a single-institution review. Arch Med Sci Atheroscler Dis. 2019;4:e89-93. https://doi.org/10.5114/amsad.2019.85380

10. Arem $\mathrm{H}$, Irwin ML. Obesity and endometrial cancer survival: a systematic review. Int J Obes (Lond). 2013;37(5):634-9. https://doi.org/10.1038/ijo.2012.94

11. Shachar SS, Williams GR, Muss HB, Nishijima TF. Prognostic value of sarcopenia in adults with solid tumours: a metaanalysis and systematic review. Eur J Cancer. 2016;57:58-67. https://doi.org/10.1016/j.ejca.2015.12.030

12. Portal D, Hofstetter L, Eshed I, Dan-Lantsman C, Sella T, Urban $\mathrm{D}$, et al. $\mathrm{L} 3$ skeletal muscle index (L3SMI) is a surrogate marker of sarcopenia and frailty in non-small cell lung cancer patients. Cancer Manag Res. 2019;11:2579-88. https://doi. org/10.2147/CMAR.S195869 
13. Ratnayake CB, Loveday BP, Shrikhande SV, Windsor JA, Pandanaboyana S. Impact of preoperative sarcopenia on postoperative outcomes following pancreatic resection: a systematic review and meta-analysis. Pancreatology. 2018;18(8):996-1004. https://doi.org/10.1016/j. pan.2018.09.011

14. Carneiro IP, Mazurak VC, Prado CM. Clinical Implications of sarcopenic obesity in cancer. Curr Oncol Rep. 2016;18(10):62. https://doi.org/10.1007/s11912-016-0546-5
15. Ni P, Marino M, Dore E, Sonis L, Ryan CM, Schneider JC, et al. Extreme response style bias in burn sunvivors. PLoS One. 2019;14(5):e0215898. https://doi.org/10.1371/journal.pone.0215898

16. Jang M, Park HW, Huh J, Lee JH, Jeong YK, Nah $Y W$, et al. Predictive value of sarcopenia and visceral obesity for postoperative pancreatic fistula after pancreaticoduodenectomy analyzed on clinically acquired CT and MRI. Eur Radiol. 2019;29(5):2417-25. https://doi. org/10.1007/s00330-018-5790-7 